\title{
Epithelioid Schwannoma of a Spinal Nerve Root
}

\author{
Rachael A. Vaubel, Howard T. Chang, Karen Fritchie, Patrice C. Abell Aleff, Mark E. Jentoft
}

Keywords: Epithelioid, myxoid schwannoma, peripheral nerve sheath tumors, schwannoma

doi:10.1017/cjn.2015.340

Can J Neurol Sci. 2016; 43: 430-433

Even though the diagnosis of schwannoma is usually straightforward in lesions with classic features, tumors with predominantly epithelioid morphology or marked myxoid change may present a diagnostic challenge. There are relatively few reported cases of epithelioid schwannoma entirely lacking classic morphology, and these have occurred in subcutaneous tissues, mucosal surfaces, or as gastrointestinal polyps. Within the spinal cord, caution must be taken to avoid misclassification of this entity as a mesenchymal neoplasm or malignant peripheral nerve sheath tumor, more commonly present with epithelioid morphology.

\section{Case Report}

The patient, a 50-year-old Caucasian woman, presented with low back pain of 5 months' duration, associated with bilateral leg pain, numbness, and weakness. Magnetic resonance imaging (MRI) revealed enlargement of a known thoracic spine tumor, which had been present for approximately 6 years. The patient had previously declined surgery because of other medical concerns; however, her symptoms had worsened. MRI now demonstrated a large, right-sided, dumbbell-shaped tumor, eroding the T6 vertebral body and extending into the neural foramen (Figure 1). The patient underwent a gross total resection. The surgical specimen was composed of pale pink, gelatinous mucoid material (Figure 2) with associated cartilage and bone. Frozen section interpretation favored a chondroid lesion with a differential diagnosis, including chordoma and myxoid chondrosarcoma (Figure 2).

On microscopic examination of the paraffin sections (Figure 3), the lesion demonstrated a proliferation of epithelioid cells in an abundant myxoid background with focal spindled cell morphology. Rare, thick-walled vessels were identified throughout the lesion and focal dense fibrous tissue, suggesting a fibrous capsule was present in some blocks. Mitotic figures were difficult to find, and no tumor necrosis was identified. No chondroid matrix production was appreciated.

Immunohistochemical stains were performed for S100, glial fibrillary acidic protein (GFAP), collagen 4 (COL4), cytokeratin (AE1/AE3, Cam5.2), epithelial membrane antigen (EMA), brachyury, HMB45, Melan A, FLI-1, and CD31. The neoplastic cells demonstrated strong nuclear and cytoplasmic expression of S100 and membranous expression of COL4 (Figure 3). Some cells appeared focally and weakly positive for GFAP. Although the histologic appearance of the lesion and S100 staining raised the possibility of chordoma, the neoplastic cells were negative for cytokeratins (AE1/AE3, Cam5.2), EMA, and brachyury as well as for HMB45, Melan A, FLI-1, and CD31.

To further characterize this lesion, electron microscopy was performed from tissue initially fixed in formalin and subsequently fixed in glutaraldehyde. The lesion demonstrated characteristic features of Schwann cell differentiation, including a continuous basement membrane, formation of mesaxons, and extracellular long-spacing collagen (Figure 4). These overall features were consistent with epithelioid schwannoma. The patient made an uneventful recovery, with resolution of her symptoms, and is disease free at 1-year follow-up.

\section{Materials ANd Methods}

Immunohistochemistry was performed using commercially available antibodies to Collagen IV, CD31, GFAP, HMB45, Melan-A (Dako; Carpinteria, CA), S100, cytokeratin AE1/AE3, Ki67 (Ventana; Tucson, AZ), brachyury (Santa Cruz; Dallas, TX), FLI-1, and Cam 5.2 (BD Pharmingen; San Jose, CA). Fixed tissue was processed for electron microscopy, embedded in epoxy resin, cut for light microscopy sections, and subsequently for ultrathin sections using an ultramicrotome. Ultrathin sections were cut at $100 \mathrm{~nm}$, mounted on copper grids, stained differentially using lead citrate, and examined in an FEI Tecnai $G^{2} 12$ transmission electron microscope. Digital images were captured with an AMT side mount CCD camera system.

\section{Discussion}

Schwannomas with epithelioid morphology are rare with only a small number of cases reported in the literature. Since the initial description in 1981, 39 cases of schwannoma with purely epithelioid morphology have been described in the English-language literature. Additional cases of schwannoma with epithelioid morphology have been reported; however, these lesions also demonstrated areas of more conventional schwannoma histology, making the diagnosis more straightforward. The majority of epithelioid schwannoma (26 cases) occurred in the cutaneous, subcutaneous, and deep soft tissues. An additional 10 cases have been reported in the colonic mucosa, presenting as polyps. ${ }^{1,2}$

From the Department of Laboratory Medicine and Anatomic Pathology, Mayo Clinic, Rochester, MN; Sparrow Health System, Lansing, MI; Electron Microscopy, Mayo Clinic, Rochester, MN.

Received March 11, 2015. Final Revisions Submitted August 27, 2015. Correspondence to: Mark E. Jentoft, Mayo Clinic, Department of Laboratory Medicine and Anatomic Pathology, 200 First Street, SW, Rochester, MN 55905. Email: jentoft.mark@mayo.edu 


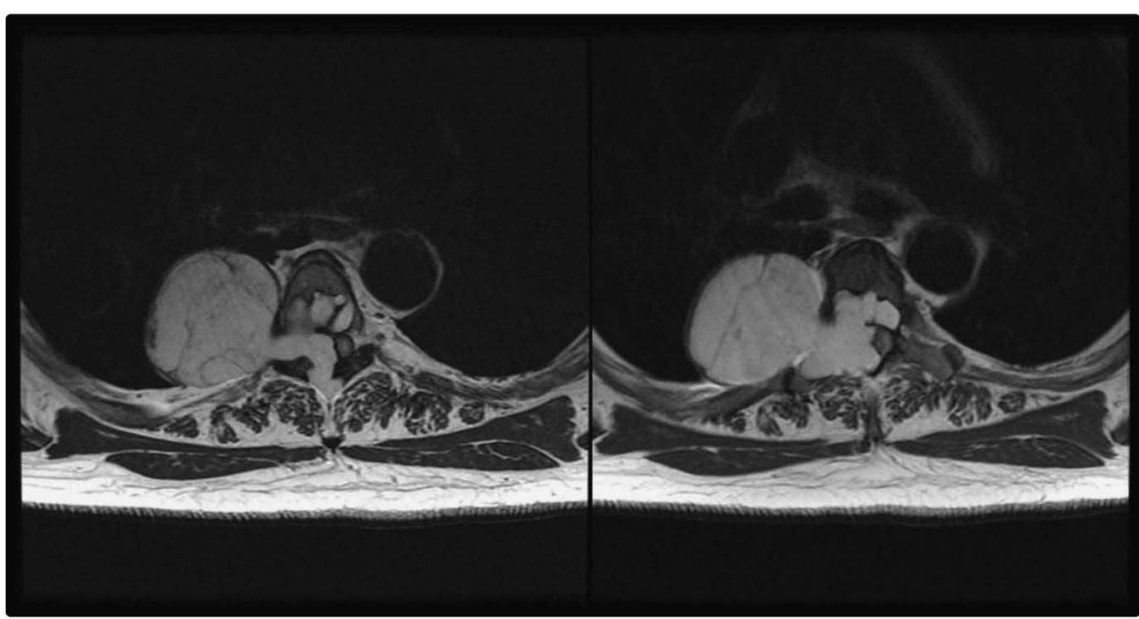

Figure 1: MRI imaging. T1 MRI demonstrated a dumbbell-shaped spinal cord lesion, eroding the $T 5$ vertebral body and extending into the neural foramen.

Intriguingly, epithelioid morphology appears to occur frequently in the colon; in one study, 4 of 20 cases of colorectal schwannoma demonstrated epithelioid morphology. ${ }^{2}$ Two additional cases have been reported in the bladder mucosa ${ }^{1}$ and a single case within the intraparotid facial nerve. To our knowledge, this case is the first epithelioid schwannoma reported in a spinal nerve root.

Epithelioid schwannomas present a diagnostic challenge because these lesions lack the typical Antoni A and B growth patterns of conventional schwannoma. However, they share a characteristic immunohistochemical staining profile that includes strong expression of $\mathrm{S} 100$ protein, membranous expression of COL4, and variable expression of other Schwann cell markers, including GFAP. The spinal location and abundant myxoid matrix in this case presented a particular diagnostic challenge and a broad differential diagnosis that included chordoma and other chondromyxoid lesions, such as a chondrosarcoma with myxoid change and extraskeletal myxoid chondrosarcoma. In contrast to chordoma, epithelioid schwannomas do not express cytokeratins, EMA, or brachyury. Even though chondrosarcoma may harbor myxoid change, areas of chondroid matrix can typically be found. Additionally, the radiologic appearance of both chordoma and chondrosarcoma would be that of a primary bone neoplasm. Extraskeletal myxoid chondrosarcoma is a distinct mesenchymal neoplasm composed of epithelioid cells arranged in cords in a myxoid background, but usually lacks the strong and diffuse S100 immunoreactivity of schwannomas. Additionally, these neoplasms are characterized by recurrent chromosomal translocations, most commonly a balanced translocation of the EWSR1 gene at 22q12 with the NR4A3 gene (alias NOR1) at 9q22, which is present in approximately $75 \%$ of cases. ${ }^{3}$ Finally, ultrastructural features of Schwann cell differentiation, including a continuous basement membrane, long-spacing collagen, and formation of mesoaxons, are helpful to exclude other neoplasms when the diagnosis is in doubt.

Epithelioid morphology has been more commonly reported in malignant peripheral nerve sheath tumors (MPNST) than in their benign counterparts. In some cases, the distinction between epithelioid MPNST and epithelioid schwannoma may be challenging, and well-defined criteria are lacking. A recent series of 63 epithelioid MPNSTs suggested that the presence of diffuse
A

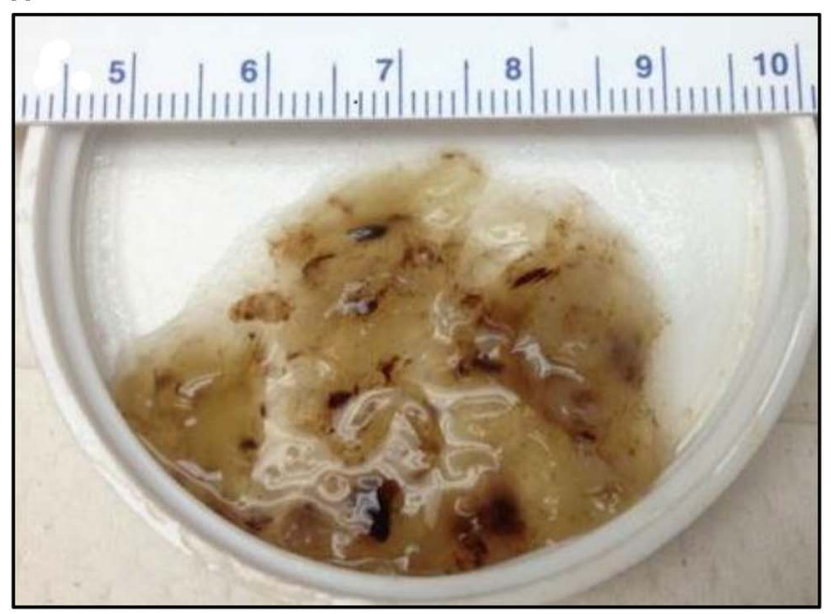

B

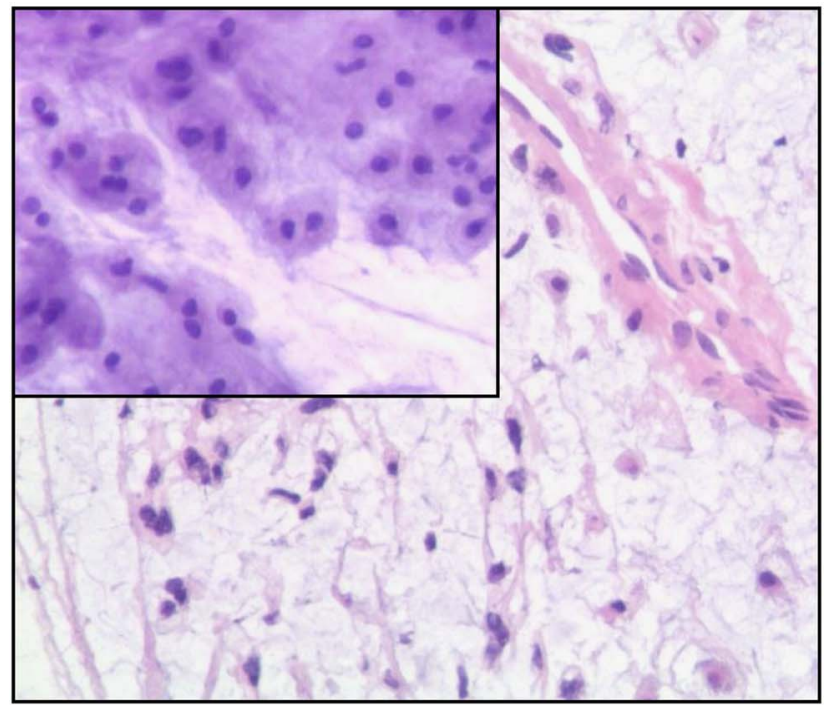

Figure 2: (A) Gross pathology. The fresh mucoid gelatinous tumor was lightly pink and translucent, but turned tan-brown after formalin fixation. (B) Touch-prep and frozen section. Touch-prep (inset) and frozen sections showed a mucoid epithelioid-chondroid appearing lesion $(400 \times$ magnification $)$. 


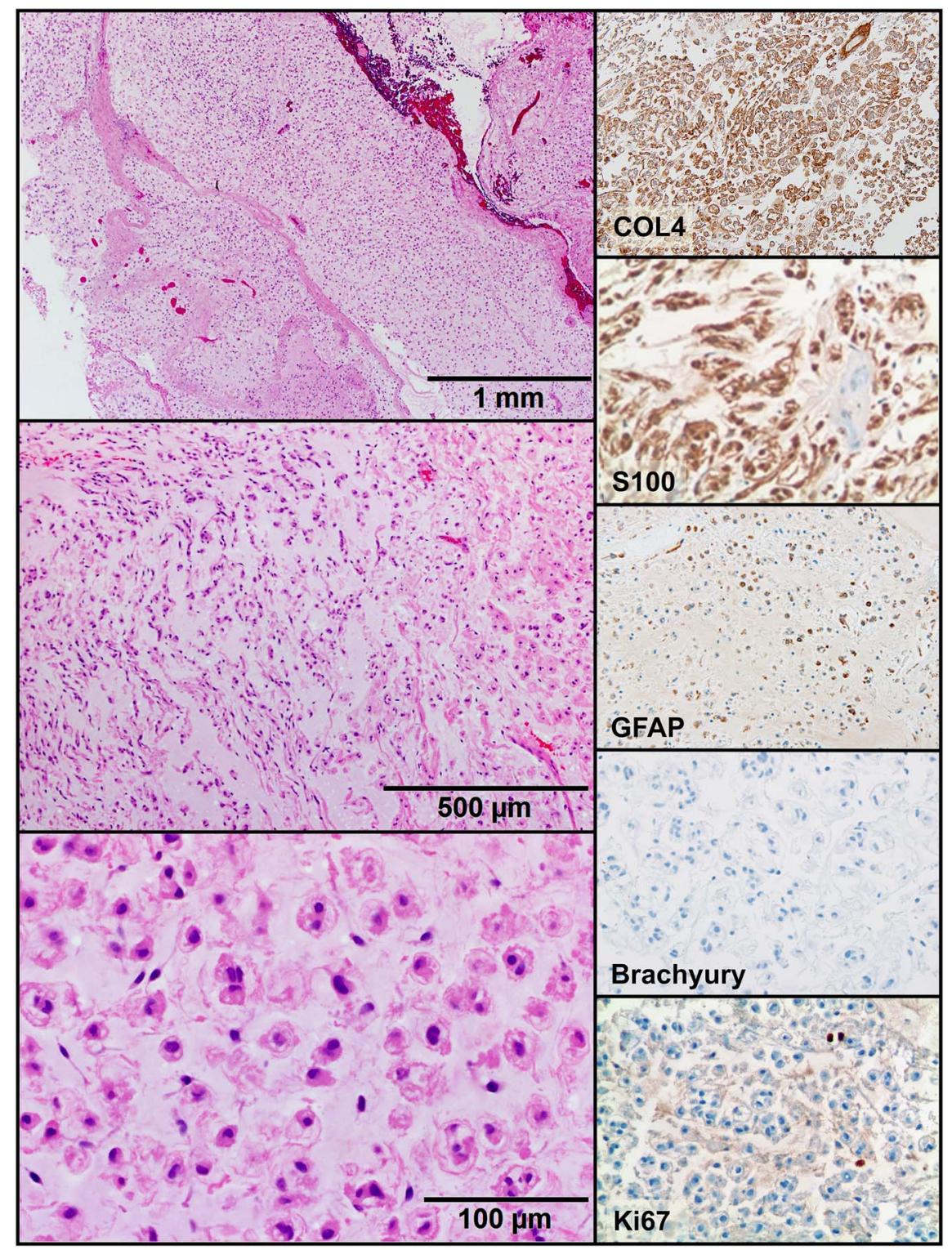

Figure 3: Tumor histology. Histologic examination revealed epithelioid cells in an abundant myxoid stroma $(H \& E, 40 \times$ top, $100 \times$ middle, $400 \times$ bottom). The tumor cells stained positive for COLA, S100, and GFAP, and negative for brachyury and cytokeratin (not shown). Ki67 had a low level of labelling $(200 \times$ magnification $)$.

nuclear atypia and pleomorphism, vesicular nuclei, atypical mitotic figures, or necrosis favor a diagnosis of epithelioid MPNST. ${ }^{4}$ Epithelioid MPNSTs also typically had an elevated mitotic rate, with a median of 5/10 per high-powered field (range, $1-46 / 10){ }^{4}$

Although malignant transformation of schwannoma is exceedingly rare, when it does occur, the tumor often shows an epithelioid morphology. McMenamin et al have extended this observation to suggest that "epithelioid malignant change," characterized by foci of epithelioid morphology arising within schwannoma with conventional architecture, may be a precursor to malignant transformation. ${ }^{5}$ However, schwannoma with purely epithelioid morphology (lacking areas of classic schwannoma morphology) appear to be a separate entity characterized by a benign clinical course. These lesions are typically slow-growing. Surgical resection of epithelioid schwannoma is almost universally curative. In the large case series of benign epithelioid peripheral nerve sheath tumors reported by Laskin et al, only a single case recurred 10 years after the initial resection, and the patient was disease free after reexcision. ${ }^{6}$ No additional recurrences have been reported in the literature.

Because schwannoma of the spinal cord/spinal nerve roots is common, epithelioid schwannoma may be an underrecognized entity in this location. This lesion may mimic malignant mesenchymal tumors, and its correct identification is essential. 


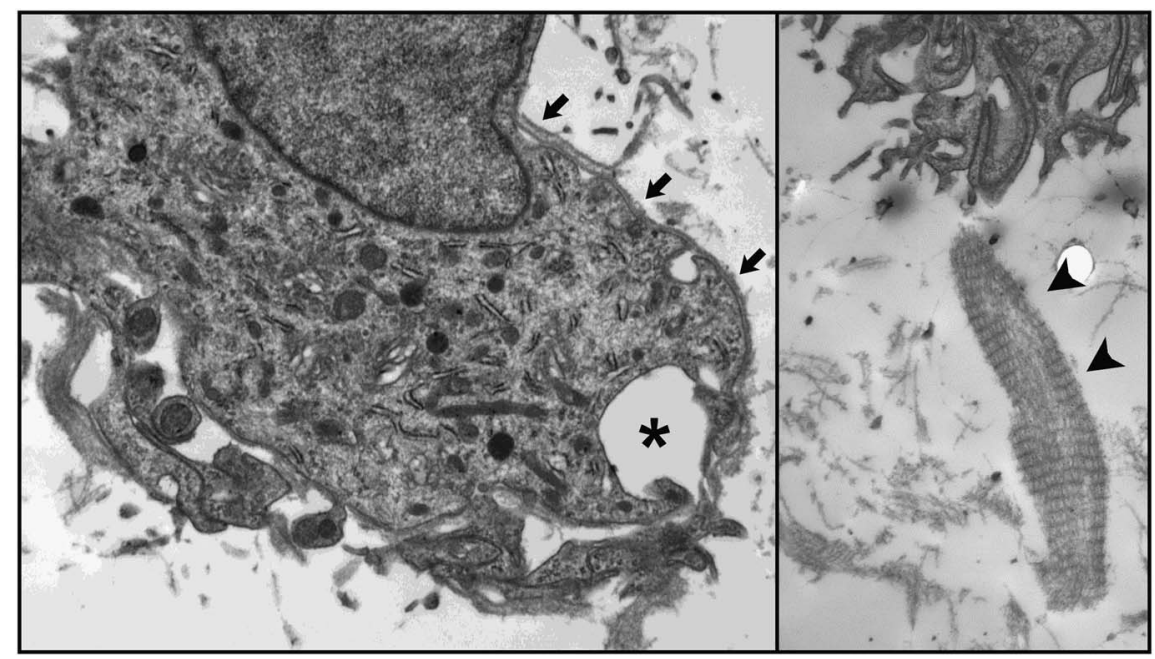

Figure 4: Ultrastructure. Electron microscopy demonstrated characteristic features of Schwann cells, including continuous basement membrane (arrows), mesaxon formation (*), and long spacing collagen (arrowheads).

\section{DisCLOSURES}

$\mathrm{RV}, \mathrm{HC}, \mathrm{KF}, \mathrm{PCAA}$, and MJ do not have anything to disclose.

\section{REFERENCES}

1. Lewin MR, Dilworth HP, Abu Alfa AK, Epstein JI, Montgomery E. Mucosal benign epithelioid nerve sheath tumors. Am J Surg Pathol. 2005;29:1310-5.

2. Miettinen M, Shekitka KM, Sobin LH. Schwannomas in the colon and rectum: a clinicopathologic and immunohistochemical study of 20 cases. Am J Surg Pathol. 2001;25:846-55.

3. Benini S, Cocchi S, Gamberi G, et al. Diagnostic utility of molecular investigation in extraskeletal myxoid chondrosarcoma. J Mol Diagn. 2014;16:314-23.
4. Jo VY, Fletcher CD. Epithelioid malignant peripheral nerve sheath tumor: clinicopathologic analysis of 63 cases. Am J Surg Pathol. 2015;39:673-82.

5. McMenamin ME, Fletcher CD. Expanding the spectrum of malignant change in schwannomas: epithelioid malignant change, epithelioid malignant peripheral nerve sheath tumor, and epithelioid angiosarcoma: a study of 17 cases. Am J Surg Pathol. 2001;25:13-25.

6. Laskin WB, Fetsch JF, Lasota J, Miettinen M. Benign epithelioid peripheral nerve sheath tumors of the soft tissues: clinicopathologic spectrum of 33 cases. Am J Surg Pathol. 2005; 29:39-51. 